\title{
STABILITY OF THE FIXED POINT PROPERTY AND UNIVERSAL MAPS
}

\author{
JOSÉ M. R. SANJURJO
}

(Communicated by Dennis K. Burke)

\begin{abstract}
We give a stability condition for the fixed point property in terms of the fundamental metric and the metric of continuity introduced by $\mathrm{K}$. Borsuk. This condition is equivalent to that originally given by $\mathrm{V}$. Klee but reflects richer properties. We introduce the notion of a proximately universal map and study many of its properties. Relationships among proximately universal maps and some generalized refinable maps introduced by E. E. Grace are studied. In particular we prove that every weakly refinable map $r: X \rightarrow Y$ is proximately universal whenever $X$ has the proximate fixed point property. This generalizes a result of Grace.
\end{abstract}

\section{INTRODUCTION}

In 1961, V. Klee introduced a stability condition related to the fixed point property [13]. Roughly speaking, a compactum $X$ satisfies such a condition if for every function $f$ of $X$ into itself which is nearly continuous there is a point of $X$ which is nearly invariant under $f$. Every compactum which has this property must also have the fixed point property. However, Klee described in [13] a plane continuum in which the fixed point property persists but fails to satisfy the stability condition. On the other hand, Klee proved that every compact metric absolute retract satisfies the stability condition. This result was later improved by Ho [10] (and according to him also by Finbow) who proved that if an approximative absolute neighborhood retract in the sense of Clapp [6] possesses the fixed point property then it also satisfies the stability condition. Recently E. E. Grace obtained a number of interesting results [8] about the preservation of the stability condition by refinable maps [7] or, more generally, by two successively broader classes of maps, proximately refinable maps and weakly refinable maps [9].

Received by the editors August 6, 1987 and, in revised form, March 10, 1988.

1980 Mathematics Subject Classification (1985 Revision). Primary 54H25; Secondary 54C10.

Key words and phrases. Proximate fixed point property, refinable map, weakly refinable map, proximately universal map.

Partially supported by CAICYT.

This paper was written while the author was visiting the Department of Mathematics of the University of Manchester. The author wishes to express his gratitude to the members of the Department for their hospitality. 
In this paper we present further contributions to the study of the compact metric spaces which have the proximate fixed point property (i.e. which satisfy the stability condition). We show that if a compactum $X$ has the proximate fixed point property then it satisfies a stability condition which is stronger than that described by Klee. More concretely, we see that compacta which are sufficiently close to $X$ in the fundamental metric introduced by Borsuk [2] (and hence in the metric of continuity) still have the $\varepsilon$-fixed point property for $\delta$ functions, with $\delta$ sufficiently small. We also prove that if a compactum $X$ is $\mathscr{P}$-like, where $\mathscr{P}$ is a class of compacta with the proximate fixed point property, then $X$ has the fixed point property for nearly extendable maps [3]. Examples of Borsuk [1] and Grace [8] show that being $\mathscr{P}$-like does not assure having the fixed point property. The fixed point property for nearly extendable maps is preserved by continuously weakly refinable maps [8] but not by refinable maps. We introduce in the paper the class of proximately universal maps and study many of its properties. This class of maps results from imposing a stability condition upon the universal maps introduced by Holsztynski [11]. We prove that a weakly refinable map $r: X \rightarrow Y$ is proximately universal whenever $X$ has the proximate fixed point property. This improves Grace's Theorem 1 in [8]. We also see, among other things, that compositions of refinable maps and proximately universal maps are always proximately universal. As a consequence, if $r: X \rightarrow Y$ is refinable and $Y$ has the proximate fixed point property, then $r$ is proximately universal.

The author wishes to express his gratitude to Fernanda Romão for her great help in the preparation of this manuscript.

\section{DEFINITIONS AND PRELIMINARIES}

Definitions [13, 14]. Let $f: X \rightarrow Y$ be a function between compacta. Let a positive number $\varepsilon$ be given. We say that $f$ is $\varepsilon$-continuous if there is a $\delta>0$ such that $d\left(f(x), f\left(x^{\prime}\right)\right)<\varepsilon$ whenever $x$ and $x^{\prime}$ are points in $X$ with $d\left(x, x^{\prime}\right)<\delta$. A compactum $X$ has the proximate fixed point property (p.f.p.p.) if for each positive number $\varepsilon$ there is a positive number $\delta$ such that every $\delta$ continuous function $f: X \rightarrow X$ has an $\varepsilon$-invariant point (i.e. a point $x$ such that $d(x, f(x))<\varepsilon)$.

Remark 1. Our definition of $\varepsilon$-continuity is slightly different from that which is usual in the literature. However, since we are dealing with compacta, this definition will help to simplify the exposition.

In our next definition we introduce the notion of a proximately universal mapping. Universal maps were first considered by Holsztynski in [11]. A map $f: X \rightarrow Y$ is called universal if for any map $g: X \rightarrow Y$ there exists $x \in X$ such that $f(x)=g(x)$. Our notion results from imposing upon universal maps a stability condition similar to that considered in the fixed point property. 
Definition. Let $f: X \rightarrow Y$ be a map between compacta. Then $f$ is called proximately universal if for every $\varepsilon>0$ there is a $\delta>0$ such that every $\delta$-continuous function $g: X \rightarrow Y$ admits a point $x \in X$ such that $d(f(x), g(x))<\varepsilon$.

As a consequence of the results in this paper it is easy to see that every map of the unit interval $I=[0,1]$ onto itself is proximately universal. However, the identity map of the Warsaw circle is an example of a map which is universal but not proximately universal.

In [2], Borsuk introduced a metrization of the hyperspace of a metric space. Borsuk's aim was to obtain a metric suitable enough to deal with shape properties of compacta. In this way he defined the fundamental metric. Let $X, Y$ be two compacta contained in an AR-space $M$. By $d_{F}(X, Y)$ we denote the infimum of the set of all positive numbers $\varepsilon$ satisfying the following condition:

There exist two fundamental sequences

$$
\underline{f}=\left\{f_{k}, X, Y\right\}_{M, M}, \quad \underline{g}=\left\{g_{k}, Y, X\right\}_{M, M}
$$

and a neighborhood $(U, V)$ of the pair $(X, Y)$ in $(M, M)$ such that for almost all $k, d\left(x, f_{k}(x)\right)<\varepsilon$ for every $x \in U$ and $d\left(y, g_{k}(y)\right)<\varepsilon$ for every $y \in V$.

Borsuk proved that the collection $2^{M}$ of all compacta lying in $M$ with the metric $d_{F}$ is a metric space. We denote this space by $2_{F}^{M}$. Borsuk also proved that for compacta $X$ and $Y$ we have $d_{F}(X, Y) \leq d_{C}(X, Y)$, where $d_{C}$ denotes the well-known metric of continuity.

Borsuk introduced in [3] a new class of maps, called nearly extendable maps, which play a significant role in connection with the Lefschetz-Hopf fixed point theorems [4]. A map $f$ of a compactum $X$ into another compactum $Y$ is said to be nearly extendable if there exist $A R$-spaces $M, N$ containing $X$ and $Y$ respectively and there is a map $\hat{f}: M \rightarrow N$ with $\hat{f}(x)=f(x)$ for every $x \in X$ such that the following condition is satisfied: For every $\varepsilon>0$ there is a neighborhood $U$ of $X$ in $M$ such that for every neighborhood $V$ of $Y$ in $N$ there is a map $g: U \rightarrow V$ with $d(\hat{f}(x), g(x))<\varepsilon$ for every $x \in U$.

Finally we recall the definition of a weakly refinable map, a notion introduced by Grace in [9]. Let $r: X \rightarrow Y$ be a map between compacta. We say that $r$ is weakly refinable if for every $\delta>0$ there is a $\delta$-continuous function $g: Y \rightarrow X$ such that $d(y, r g(y))<\delta$ for all $y \in Y$.

All the spaces considered in the paper are metric and we always use the letter $d$ to represent the corresponding distance. Compactum means compact metric space and a map is a continuous function.

\section{THE PROXIMATE FIXED POINT PROPERTY}

In our first result we give two conditions which are equivalent to the p.f.p.p. The first of them is a stability condition for the fixed point property which is stronger than that considered by Klee. 
Theorem 1. Let $X$ be a compactum lying in an AR-space $M$. Then the following statements are equivalent

(a) $X$ has the p.f.p.p.

(b) For every $\varepsilon>0$ there is a $\delta>0$ such that if $Y$ is a compactum in $M$ with $d_{F}(X, Y)<\delta$, then every $\delta$-continuous function $g: Y \rightarrow Y$ has an $\varepsilon$-invariant point.

(c) For every $\varepsilon>0$ there is a compactum $Y$ and $a \delta>0$ such that $d_{F}(X, Y)<\varepsilon$ and every $\delta$-continuous function $g: Y \rightarrow Y$ has an $\varepsilon$ invariant point.

Proof. We shall only prove that (a) implies (b) and that (c) implies (a) since the converse implications are obvious. Let $\varepsilon>0$ be given and consider $\eta>0$ such that every $\eta$-continuous function from $X$ into $X$ has an $\varepsilon / 5$-invariant point. Let $\delta=\min \{\eta / 5, \varepsilon / 5\}$ and consider a $\delta$-continuous function $g: Y \rightarrow Y$ where $Y$ is a compactum such that $d_{F}(X, Y)<\delta$. Then there is a positive $\delta^{\prime}<\delta$ such that $d\left(g(y), g\left(y^{\prime}\right)\right)<\delta$ whenever $y$ and $y^{\prime}$ are points of $Y$ with $d\left(y, y^{\prime}\right)<\delta^{\prime}$. Let $U$ and $V$ be neighborhoods in $M$ of $X$ and $Y$ respectively such that $d(x, X)<\delta^{\prime} / 3$ for every $x \in U$ and $d(y, Y)<\delta^{\prime} / 3$ for $y \in V$. Since $d_{F}(X, Y)<\delta$ there are maps $h: X \rightarrow V$ and $l: Y \rightarrow U$ with $d(x, h(x))<\delta$ for $x \in X$ and $d(y, l(y))<\delta$ for $y \in Y$. We can also define functions $\alpha: U \rightarrow X$ and $\beta: V \rightarrow Y$ such that $d(\alpha(x), x)<\delta^{\prime} / 3$ for $x \in U$ and $d(\beta(y), y)<\delta^{\prime} / 3$ for $y \in V$. Obviously $\alpha$ and $\beta$ are $\delta^{\prime}$. continuous and, as a consequence, $h^{\prime}=\beta h$ and $l^{\prime}=\alpha l$ also are $\delta^{\prime}$-continuous. It is easy to see that $d\left(h^{\prime}(x), x\right)<2 \delta$ for $x \in X$ and $d\left(l^{\prime}(y), y\right)<2 \delta$ for $y \in Y$. Since $h^{\prime}$ is $\delta^{\prime}$-continuous we have that $g h^{\prime}$ is $\delta$-continuous. Hence, there is a $\mu>0$ such that $d\left(g h^{\prime}(x), g h^{\prime}\left(x^{\prime}\right)\right)<\delta$ whenever $d\left(x, x^{\prime}\right)<\mu$. Therefore if $d\left(x, x^{\prime}\right)<\mu$ then

$$
\begin{aligned}
& d\left(l^{\prime} g h^{\prime}(x), l^{\prime} g h^{\prime}\left(x^{\prime}\right)\right) \leq d\left(l^{\prime} g h^{\prime}(x), g h^{\prime}(x)\right) \\
& \quad+d\left(g h^{\prime}(x), g h^{\prime}\left(x^{\prime}\right)\right)+d\left(g h^{\prime}\left(x^{\prime}\right), l^{\prime} g h^{\prime}\left(x^{\prime}\right)\right)<2 \delta+\delta+2 \delta \leq \eta .
\end{aligned}
$$

Hence $l^{\prime} g h^{\prime}$ is a $\eta$-continuous function from $X$ into $X$ and, as a consequence, there is a point $x \in X$ such that $d\left(l^{\prime} g h^{\prime}(x), x\right)<\varepsilon / 5$. Set $y=h^{\prime}(x)$. Then we have

$$
\begin{aligned}
d(g(y), y)= & d\left(g h^{\prime}(x), h^{\prime}(x)\right) \leq d\left(g h^{\prime}(x), l^{\prime} g h^{\prime}(x)\right) \\
& +d\left(l^{\prime} g h^{\prime}(x), x\right)+d\left(x, h^{\prime}(x)\right)<2 \delta+\varepsilon / 5+2 \delta \leq \varepsilon .
\end{aligned}
$$

Hence $g$ admits an $\varepsilon$-invariant point and this completes the proof that (a) implies (b).

To prove that (c) implies (a) let $\varepsilon>0$ be given. Then, there is a compactum $Y$ such that $d_{F}(X, Y)<\varepsilon / 5$ and a $\delta>0$ such that every $\delta$-continuous function from $Y$ into itself has an $\varepsilon / 5$-invariant point. Since $d_{F}(X, Y)<\varepsilon / 5$ it is easy to see, by an argument similar to that used before, that there is a $\delta$ continuous function $h: X \rightarrow Y$ such that $d(h(x), x)<2 \varepsilon / 5$ for every $x \in X$. Hence there is a $\eta>0$ such that if $f: X \rightarrow X$ is $\eta$-continuous then $h f$ is 
$\delta$-continuous. Suppose that such a $\eta$-continuous function $f$ is given. We can find a function $l: Y \rightarrow X$ such that $h f l$ is $\delta$-continuous and $d(l(y), y)<2 \varepsilon / 5$ for every $y \in Y$. This is also a consequence of the fact that $d_{F}(X, Y)<\varepsilon / 5$. Hence, there is a point $y \in Y$ such that $d(h f l(y), y)<\varepsilon / 5$. Set $x=l(y)$. Then we have

$$
\begin{aligned}
d(f(x), x)= & d(f l(y), l(y) \leq d(f l(y), h f l(y)) \\
& +d(h f l(y), y)+d(y, l(y))<2 \varepsilon / 5+\varepsilon / 5+2 \varepsilon / 5=\varepsilon .
\end{aligned}
$$

Hence every $\eta$-continuous function $f: X \rightarrow X$ has an $\varepsilon$-invariant point and, as a consequence, $X$ has the p.f.p.p. This completes the proof of the theorem.

Corollary 1. A compactum $X$ lying in $M \in A R$ has the p.f.p.p. if and only if for every $\varepsilon>0$ there is a $\delta>0$ such that if $Y$ is a compactum in $M$ with $d_{C}(X, Y)<\delta$ then every $\delta$-continuous function $g: Y \rightarrow Y$ has an $\varepsilon$-invariant point.

Corollary 2. The set of all compacta in $M$ having the p.f.p.p. is closed in $2_{F}^{M}$.

Remark 2. Borsuk's Example 4.3 in [2] shows that the subset of the hyperspace $2_{F}^{M}$ consisting of all compacta with the fixed point property is, in general, not closed. Čerin and Šostak have proved in [5], Theorem 3.3], the analogue of Corollary 2 for the metric of continuity.

Grace proved in [8] that if $\mathscr{P}$ is a class of compacta with the p.f.p.p. and $X$ is a compactum weakly refinably $\mathscr{P}$-like then $X$ also has the p.f.p.p. However, if we omit the condition of weak refinability then Grace's proposition is no longer true. Borsuk described in [1] a continuum $X$ which is quasi-homeomorphic to a cube but $X$ does not have the fixed point property. Grace gave in [8] an example of a refinable, continuously weakly refinable map from a continuum that does not have the f.p.p. onto a continuum with the p.f.p.p. Hence in both cases $X$ is like a continuum with the p.f.p.p. but $X$ does not have the fixed point property. In the next proposition we prove that if $X$ is $\mathscr{P}$-like (where $\mathscr{P}$ is a class of compacta with the p.f.p.p.) then $X$ has the fixed point property for the class of nearly extendable maps introduced by $\mathrm{K}$. Borsuk.

Theorem 2. Let $\mathscr{P}$ be a class of compacta with the p.f.p.p. and let $X$ be a compactum. If $X$ is $\mathscr{P}$-like then every nearly extendable map from $X$ into itself has a fixed point.

Proof. We can assume that $X$ lies in the Hilbert cube, $Q$. Let $h: X \rightarrow X$ be a nearly extendable map and consider a map $\hat{h}: Q \rightarrow Q$ such that $\hat{h}(x)=h(x)$ for every $x \in X$. Let $\varepsilon>0$ be given. Then, there is a $\eta>0, \eta<\varepsilon / 3$, such that $d\left(\hat{h}(x), \hat{h}\left(x^{\prime}\right)\right)<\varepsilon / 3$ whenever $x$ and $x^{\prime}$ are points in $Q$ with $d\left(x, x^{\prime}\right)<\eta$. Since $h$ is nearly extendable there is a neighborhood $U$ of $X$ in $Q$ such that for every neighborhood $U^{\prime}$ of $X$ in $Q$ there is a map $h^{\prime}: U \rightarrow U^{\prime}$ with $d\left(h^{\prime}(x), \hat{h}(x)\right)<\varepsilon / 12$ for every $x \in U$. We can assume that $d(x, X)<\eta$ for every $x \in U$. Since $X$ is $\mathscr{P}$-like there is a compactum $Y \in \mathscr{P}$ and maps $f: X \rightarrow Y, g: Y \rightarrow U$ such that $d(g f(x), x)<\varepsilon / 12$ for every $x \in X$ 
(see [15], p. 27, Theorem 9]). Select $\mu>0$ such that $d\left(g(y), g\left(y^{\prime}\right)\right)<\varepsilon / 12$ whenever $d\left(y, y^{\prime}\right)<\mu$ and consider a $\mu^{\prime}>0$ such that every $\mu^{\prime}$-continuous function from $Y$ into itself has a $\mu$-invariant point. An argument similar to that used in the proof of Theorem 1 shows that there is a neighborhood $U^{\prime}$ of $X$ in $Q$ and a function $\alpha: U^{\prime} \rightarrow X$ such that $d(\alpha(x), x)<\varepsilon / 12$ for $x \in U^{\prime}$ and $f \alpha$ is $\mu^{\prime}$-continuous. Consider now a map $h^{\prime}: U \rightarrow U^{\prime}$ such that $d\left(h^{\prime}(x), \hat{h}(x)\right)<\varepsilon / 12$ for $x \in U$. Then $f \alpha h^{\prime} g$ is $\mu^{\prime}$-continuous and, in consequence, there is a point $y \in Y$ such that $d\left(f_{\alpha} h^{\prime} g(y), y\right)<\mu$. Hence

$$
d\left(g f \alpha h^{\prime} g(y), g(y)\right)<\varepsilon / 12
$$

and

$$
\begin{gathered}
d\left(h^{\prime} g(y), g(y)\right) \leq d\left(h^{\prime} g(y), \alpha h^{\prime} g(y)\right)+d\left(\alpha h^{\prime} g(y), g f \alpha h^{\prime} g(y)\right) \\
+d\left(g f \alpha h^{\prime} g(y), g(y)\right)<3 \varepsilon / 12 .
\end{gathered}
$$

This implies that

$$
\begin{aligned}
d(\hat{h} g(y), g(y)) & \leq d\left(\hat{h} g(y), h^{\prime} g(y)\right)+d\left(h^{\prime} g(y), g(y)\right) \\
& <\varepsilon / 12+3 \varepsilon / 12=\varepsilon / 3 .
\end{aligned}
$$

Select now a point $x \in X$ such that $d(x, g(y))<\eta$. Then

$$
d(h(x), x) \leq d(h(x), \hat{h} g(y))+d(\hat{h} g(y), g(y))+d(g(y), x)<\varepsilon .
$$

Hence for every $\varepsilon<0$ there is a point which is $\varepsilon$-invariant under $h$ and this implies that $h$ has a fixed point. This completes the proof of the theorem.

The author does not know whether the p.f.p.p. can be replaced in Theorem 2 by the weaker assumption of f.p.p.

The fact that a compactum $X$ has the fixed point property for nearly extendable maps is not preserved by refinable maps: Grace gives in [8] an example of a refinable map from a continuum with the f.p.p. onto the circle. We have however the following result whose proof is left to the reader.

Theorem 3. If $f: X \rightarrow Y$ is a continuously weakly refinable map between compacta [8] and $X$ has the fixed point property for nearly extendable maps then also does $Y$.

In the sequel, we are going to study some of the basic properties of proximately universal maps. Later we shall establish some relations among this kind of maps and refinable maps.

Theorem 4. Let $f: X \rightarrow Y$ be a map of compacta. Then,

(a) If $f$ is proximately universal then $f$ is universal.

(b) If $f$ is proximately universal then $f(X)=Y$ and $Y$ has the p.f.p.p.

(c) $X$ has the p.f.p.p. if and only if $\mathrm{id}_{X}$ is proximately universal.

(d) Consider a map $g: Y \rightarrow Z$. If $g f$ is proximately universal then so is $g$. 
(e) Let $A$ be a closed subset of $X$. If the restriction $f_{\mid A}: A \rightarrow Y$ is proximately universal then so is $f$.

(f) Suppose $h: X^{\prime} \rightarrow X$ and $g: Y \rightarrow Y^{\prime}$ are homeomorphisms and $f$ is proximately universal. Then $g f h$ is proximately universal.

(g) The set of all proximately universal maps from $X$ into $Y$ is closed in $Y^{X}$ (with the topology of uniform convergence).

(h) If $Y$ is an approximative absolute neighborhood retract in the sense of Clapp then $f$ is proximately universal if and only if $f$ is universal.

(i) If $X$ is connected, $Y=[0,1]$ and $f$ is onto, then $f$ is proximately universal.

Proof. (h) is a consequence of the fact, proved by Ho [10], that if $Y \in$ AANR $_{C}$ then for each $\varepsilon>0$ there is a $\delta>0$ such that every $\delta$-continuous function $g: X \rightarrow Y$ has a continuous $\varepsilon$-approximation. The rest of the proof is left to the reader.

We now quote another result of Ho [10] from which we can derive a characterization of proximately universal maps when we consider compacta embedded in the Hilbert cube.

Lemma 1 ([Ho]). Let $X$ be a paracompact topological space and $V$ be a normed linear space. Then each $\delta$-continuous function $f: X \rightarrow V$ has a continuous $\delta$ approximation.

Ho's lemma is also valid when $V$ is the Hilbert cube.

Our next result can be proved without effort with the help of Ho's lemma. We leave its proof to the reader.

Theorem 5. Let $f: X \rightarrow Y$ be a map of compacta, where $Y$ lies in the Hilbert cube, $Q$. Then $f$ is proximately universal if and only if for every $\varepsilon>0$ there is a neighborhood $V$ of $Y$ in $Q$ such that for every map $g: X \rightarrow V$ there is a point $x \in X$ with $d(f(x), g(x))<\varepsilon$.

Corollary 3. Let $X$ be a compactum lying in the Hilbert cube, $Q$. Then $X$ has the p.f.p.p. if and only if for every $\varepsilon>0$ there is a neighborhood $U$ of $X$ in $Q$ such that for every map $f: X \rightarrow U$ there is a point $x \in X$ with $d(f(x), x)<\varepsilon$.

The advantage of Theorem 5 and Corollary 3 is that they allow us to replace $\delta$-continuous functions by maps. In fact, they also represent a kind of stability condition because they state that maps taking values outside, but very close to, our compacta still have $\varepsilon$-fixed points.

Ho's lemma can be also used to give a characterization of the class of weakly refinable maps introduced by Grace [9]. If $r: X \rightarrow Y$ is a map between compacta lying in the Hilbert cube, $Q$, then since $Q$ is an absolute retract we may consider a map $\hat{r}: Q \rightarrow Q$ such that $\hat{r}(x)=r(x)$ for every $x \in X$. Then we have the following result whose proof is omitted.

Theorem 6. Let $r: X \rightarrow Y$ be a map of compacta, where $X$ and $Y$ lie in the Hilbert cube $Q$. Then $r$ is weakly refinable if and only if for every $\delta>0$ there 
is a map $h: Y \rightarrow U$ (where $U$ is the $\delta$-neighborhood of $X$ in $Q$ ) such that $d(\hat{r} h(y), y)<\delta$ for every $y \in Y$.

In our next result we establish a relation between the notion of a weakly refinable map and the notion of a proximately universal map. This result improves Grace's Theorem 1 in [8] and it can be proved following the pattern of Grace's proof. However we give a different kind of proof which makes use of our Theorem 6.

Theorem 7. Let $r: X \rightarrow Y$ be a weakly refinable map of compacta and suppose $X$ has the p.f.p.p., then $r$ is proximately universal.

Proof. We can assume that $X$ and $Y$ lie in the Hilbert cube $Q$. Consider a map $\hat{r}: Q \rightarrow Q$ such that $\hat{r}(x)=r(x)$ for every $x \in X$. Let $\varepsilon>0$ be given and select a $\delta>0$ such that $d\left(\hat{r}(x), \hat{r}\left(x^{\prime}\right)\right)<\varepsilon / 2$ whenever $d\left(x, x^{\prime}\right)<\delta$. By Corollary 3 there is a neighborhood $W$ of $X$ in $Q$ such that every map $f: X \rightarrow W$ has a $\delta$-invariant point. By using Theorem 6 it is easy to see that there are neighborhoods $U$ and $V$ of $X$ and $Y$ respectively in $Q$ (with $U \subset$ $W)$ and a map $h: V \rightarrow U$ such that $d(\hat{r} h(y), y)<\varepsilon / 2$ for $y \in V$. Consider a map $g: X \rightarrow V$. Then, there is an $x \in X$ such that $d(h g(x), x)<\delta$ and, as a consequence, $d(\hat{r} h g(x), \hat{r}(x))<\varepsilon / 2$.

Hence $d(g(x), r(x)) \leq d(g(x), \hat{r} h g(x))+d(\hat{r} h g(x), r(x))<\varepsilon$ which proves that $r$ is proximately universal.

Corollary 4 (Grace). Suppose $r: X \rightarrow Y$ is weakly refinable and $X$ has the p.f.p.p. Then $Y$ has the p.f.p.p.

Proof. It is an immediate consequence of Theorem 4, (b) and Theorem 7.

In our next theorem we study the behaviour of the composition of refinable maps and proximately universal maps. It should be noted that it is not in general true that the composition of two proximately universal maps is proximately universal (Holsztynski's examples in [12] also apply in the proximate case).

Theorem 8. Let $X, Y$ and $Z$ be compacta. Then,

(a) If $f: X \rightarrow Y$ is proximately universal and $r: Y \rightarrow Z$ is weakly refinable, then $r f$ is proximately universal.

(b) If $r: X \rightarrow Y$ is refinable and $f: Y \rightarrow Z$ is proximately universal, then $f r$ is proximately universal.

Proof. Part (a) is a generalization of Theorem 7 and its proof is omitted, we shall prove Part (b). Let $\varepsilon>0$ be given and consider a $\delta>0, \delta<\varepsilon / 3$, such that for every $\delta$-continuous function $h: Y \rightarrow Z$ there is a point $y \in$ $Y$ with $d(h(y), f(y))<\varepsilon / 3$. Let $g: X \rightarrow Z$ be a $\delta$-continuous function and select a $\delta^{\prime}>0$ such that $d\left(g(x), g\left(x^{\prime}\right)\right)<\delta$ and $d\left(f(x), f\left(x^{\prime}\right)\right)<\varepsilon / 3$ whenever $d\left(x, x^{\prime}\right)<\delta^{\prime}$. Since $r$ is refinable it is not difficult to see that there is a surjective map $r^{\prime}: X \rightarrow Y$ with $d\left(r, r^{\prime}\right)<\delta^{\prime}$ and a $\delta^{\prime}$-continuous function $h: Y \rightarrow X$ such that $d\left(h r^{\prime}, \mathrm{id}_{X}\right)<\delta^{\prime}$. Then $g h$ is a $\delta$-continuous 
function from $Y$ into $Z$ and, in consequence, there is a point $y \in Y$ such that $d(f(y), g h(y))<\varepsilon / 3$. Select a point $x \in X$ such that $r^{\prime}(x)=y$, then

$$
\begin{aligned}
d(f r(x), g(x)) \leq & d\left(f r(x), f r^{\prime}(x)\right)+d\left(f r^{\prime}(x), g h r^{\prime}(x)\right) \\
& +d\left(g h r^{\prime}(x), g(x)\right)<\varepsilon .
\end{aligned}
$$

Hence $f r$ is proximately universal and the proof of the theorem is complete.

Corollary 5. Suppose $r: X \rightarrow Y$ is a refinable map and $Y$ has the p.f.p.p. Then $r$ is proximately universal.

Proof. It is an immediate consequence of Theorem 4, (c) and Theorem 8.

Our next result gives a relation between the proximate universality of the product of the mappings and the proximate universality of the composition of the same mappings. This product criterion of proximate universality is a proximate version of a theorem of Holsztynski [12].

Theorem 9. Let $f_{1}: X_{1} \rightarrow X_{2}$ and $f_{2}: X_{2} \rightarrow X_{3}$ be mappings of compacta. If the Cartesian product $f_{1} \times f_{2}: X_{1} \times X_{2} \rightarrow X_{2} \times X_{3}$ is proximately universal, then the composition $f_{2} f_{1}: X_{1} \rightarrow X_{3}$ is also proximately universal.

Proof. We consider in $X_{1} \times X_{2}$ the formal analogue of the Euclidean distance. Then if $g: X_{1} \rightarrow X_{3}$ is a function we can define $G: X_{1} \times X_{2} \rightarrow X_{2} \times X_{3}$ by $G\left(x_{1}, x_{2}\right)=\left(x_{2}, g\left(x_{1}\right)\right)$ and for every $\delta^{\prime}>0$ there is a $\delta>0$ such that if $g$ is $\delta$-continuous then $G$ is $\delta^{\prime}$-continuous. Let $\varepsilon>0$ be given and consider an $\varepsilon^{\prime}>0, \varepsilon^{\prime}<\varepsilon / 2$, such that $d\left(f_{2}(x), f_{2}(y)\right)<\varepsilon / 2$ whenever $d(x, y)<\varepsilon^{\prime}$. Select a $\delta^{\prime}>0$ related to $\varepsilon^{\prime}$ by the proximate universality of $f_{1} \times f_{2}$. Then, if $g: X_{1} \rightarrow X_{3}$ is a $\delta$-continuous function we have that $G$ is $\delta^{\prime}$-continuous and, in consequence, there is a point $\left(x_{1}, x_{2}\right) \in X_{1} \times X_{2}$ such that $d\left(\left(f_{1}\left(x_{1}\right), f_{2}\left(x_{2}\right)\right)\right.$, $\left.\left(x_{2}, g\left(x_{1}\right)\right)\right)<\varepsilon^{\prime}$. Hence $d\left(f_{2} f_{1}\left(x_{1}\right), f_{2}\left(x_{2}\right)\right)<\varepsilon / 2$ and $d\left(f_{2}\left(x_{2}\right), g\left(x_{1}\right)\right)<$ $\varepsilon^{\prime}<\varepsilon / 2$. This implies that $d\left(f_{2} f_{1}\left(x_{1}\right), g\left(x_{1}\right)\right)<\varepsilon$ and, in consequence, $f_{2} f_{1}$ is proximately universal. This completes the proof of the theorem.

At the end of this paper [10], Ho raised the question whether an FANR satisfying the f.p.p. must also satisfy the p.f.p.p. It is well known that the Warsaw Circle (which is an FANR) has the f.p.p. but it is easy to see, with the help of our Corollary 3, that it does not have the p.f.p.p. Hence, the answer to Ho's question is negative. A second question raised by Ho is the following: What kind of spaces satisfy the conjunction of the two following properties?

(1) There is a $\delta>0$ such that any two $\delta$-close maps of $X$ are homotopic.

(2) For each $\varepsilon>0$ there is a $\delta>0$ such that each $\delta$-continuous function of $X$ has a continuous $\varepsilon$-approximation.

This question is relevant because Ho showed that a Lefschetz number can be assigned to $\delta$-continuous functions of compacta satisfying conditions (1) and (2) and this number can be used in a sufficient condition for the function to possess an $\varepsilon$-invariant point. Our next result gives an answer to Ho's question. We recall that a compactum $X$ is an absolute neighborhood homotopy retract 
[16] if there is a neighborhood $U$ of $X$ in $Q$ and a map $r: U \rightarrow X$ such that $\left.r\right|_{X} \simeq \mathrm{id}_{X}$

Theorem 10. A compactum $X$ satisfies the conjunction of properties (1) and (2) if and only if $X \in A A N R_{C} \cap A N H R$, where $A N H R$ denotes the class of absolute neighborhood homotopy retracts [16].

Proof. Ho remarks in [10] that condition (2) characterizes compact AANR ${ }_{C}$ 's. We shall prove that an AANR $C, X$, belongs to the class of ANHR's if and only if $X$ satisfies condition (1). If $X \in$ ANHR then there is a closed neighborhood $U$ of $X$ in $Q$ and a map $r: U \rightarrow X$ such that $r_{\mid X} \simeq \mathrm{id}_{X}$. We can assume that $U \in \mathrm{ANR}$ and in consequence there is a $\delta>0$ such that two $\delta$-close maps taking values in $U$ are homotopic. Consider two $\delta$-close maps $f, g: X \rightarrow X$. We have that $j f \simeq j g$, where $j: X \rightarrow U$ is the inclusion and, in consequence, $f=\mathrm{id}_{X} \cdot f \simeq r_{\mid X} \cdot f=r j f \simeq r j g=r_{\mid X} \cdot g \simeq \mathrm{id}_{X} \cdot g=g$. Conversely, if $X \in$ AANR $_{C}$ and $X$ satisfies condition (1) then there is a $\delta$ such that any two $\delta$-close maps of $X$ are homotopic and there is a neighborhood $U$ of $X$ in $Q$ and a map $r: U \rightarrow X$ with $d(r(x), x)<\delta$ for all $x \in X$. Hence $r_{\mid X} \simeq \mathrm{id}_{X}$ and $X \in \mathrm{ANHR}$.

\section{REFERENCES}

1. K. Borsuk, Sur un probleme de MM. Kuratowski et Ulam, Fund. Math. 31 (1938), 154-559.

2. __ On a metrization of the hyperspace of a metric space, Fund. Math. 94 (1977), 191-207.

3. __ On nearly extendable maps, Bull. Acad. Polon Sci. 23 (1975), 753-760.

4. __, On the Lefschetz-Hopf fixed point theorem for nearly extendable maps, Bull. Acad. Polon. Sci. 23 (1975), 1273-1279.

5. Z. Čerin and A. P. Šostak, Some remarks on Borsuk's fundamental metric, Colloq. Math. Soc. Janos Bolyai, Budapest, 1978, pp. 233-252.

6. M. H. Clapp, On a generalization of absolute neighborhood retracts, Fund. Math. 70 (1971), 117-130.

7. J. Ford and J. W. Rogers, Jr., Refinable maps, Colloq. Math. 39 (1978), 263-269.

8. E. E. Grace, Refinable maps and the proximate fixed point property, Topology Proc. 10 (1985), 293-303.

9. __ Generalized refinable maps, Proc. Amer. Math. Soc. 98 (1986), 329-335.

10. C. Ho, On a stability theorem for the fixed point property, Fund. Math. 111 (1981), 169-177.

11. W. Holsztynski, Une généralisation du théorème de Brouwer sur les points invariants, Bull. Acad. Polon Sci. 12 (1964), 603-606.

12. __ On the composition and products of universal mappings, Fund. Math. 64 (1969), 181188.

13. V. L. Klee, Stability of the fixed point property, Colloq. Math. 8 (1961), 43-46.

14. V. L. Klee and A. Yandl, Some proximate concepts in topology, Symposia Math. Publ. Inst. Naz. di Alta Matematica, Academic Press 16 (1974), 21-39.

15. K. Kuratowski, Topology, Volume II, Academic Press, New York, PWN, Warszawa, 1968.

16. C. W. Saalfrank, Neighborhood retraction generalized for compact Hausdorff spaces, Portugal Math. 20 (1961), 11-16.

Departamento de Geometría y Topología, Facultad de Matemáticas, Universidad Complutense, 28040 Madrid, Spain 